\title{
ON THE CURVATURE OF NONREGULAR SADDLE SURFACES IN THE HYPERBOLIC AND SPHERICAL THREE-SPACE
}

\author{
DIMITRIOS E. KALIKAKIS
}

Received 9 November 2001

This paper proves that any nonregular nonparametric saddle surface in a threedimensional space of nonzero constant curvature $\kappa$, which is bounded by a rectifiable curve, is a space of curvature not greater than $\kappa$ in the sense of Aleksandrov. This generalizes a classical theorem by Shefel' on saddle surfaces in $\mathbb{E}^{3}$.

\section{Introduction}

The class of saddle surfaces is dual to the class of convex surfaces. A surface in a Euclidean $n$-space is said to be a saddle surface if it is impossible to cut off a crust by any hyperplane. In contrast to the theory of convex surfaces, the results in the theory of saddle surfaces are in many respects far from complete. One of the central problems in this area is the study of the intrinsic geometry. Although it is known that the Gaussian curvature of a regular saddle surface in $\mathbb{E}^{n}$ is nonpositive, it remains an open question whether the intrinsic curvature of any nonregular saddle surface in $\mathbb{E}^{n}$ is nonpositive. An affirmative answer has been given by Shefel' $[4,7]$ when $n=2$ (for any simply connected saddle surface), and when $n=3$ (for any nonparametric saddle surface). The answer is still not known for $n>3$.

In order to describe our results on saddle surfaces, first we need to introduce some terminology. The $n$-dimensional $\kappa$-space $\mathbb{S}_{\kappa}^{n}(\kappa$-plane for $n=2)$ is the hyperbolic space $\mathbb{H}_{\kappa}^{n}$ for $\kappa<0$, the Euclidean space $\mathbb{E}^{n}$ for $\kappa=0$, and the upper open hemisphere $\mathbb{S}_{+}^{n}\left(\kappa^{-1 / 2}\right)$ of $\mathbb{E}^{n+1}$ of radius $\kappa^{-1 / 2}$ with the induced metric, when $\kappa>0$. Every $\mathbb{S}_{\kappa}^{n}$ is a Riemannian simply connected manifold of constant sectional curvature $\kappa$ such that any pair of points can be joined by a unique geodesic segment. Notice that $\mathbb{S}_{\kappa}^{n}$ is a complete space only if $\kappa \leq 0$.

A nonparametric surface in the Beltrami-Klein model of $\mathbb{H}_{\kappa}^{3}$ is a continuous function $z=f(x, y)$, provided $x^{2}+y^{2}+z^{2}<-1 / \kappa$. A nonparametric surface in 
$\mathbb{S}_{+}^{3}\left(\kappa^{-1 / 2}\right)$ is a surface represented in the form $\mathbf{r}(x, y)=\left(x / a, y / a, f(x, y) / a, \kappa^{-1 / 2} / a\right)$, where $a=\left(1+\kappa x^{2}+\kappa y^{2}+\kappa f^{2}(x, y)\right)^{1 / 2}$ and $f$ is a continuous function of two variables. The principal result of this work is the following.

THEOREM 1.1. If a nonparametric saddle surface in $\mathbb{S}_{\kappa}^{3}(\kappa \neq 0)$ is bounded by a rectifiable curve, then it is a space of curvature bounded from above by $\kappa$ in the sense of Aleksandrov.

The converse of Theorem 1.1 does not hold as Example 6.1 shows. The proof of Theorem 1.1 is based on the possibility of approximating a nonparametric saddle surface in $\mathbb{S}_{\kappa}^{3}$ by saddle polyhedra (Lemma 5.2) and on a characterization of spaces of curvature bounded from above in the sense of Aleksandrov due to Reshetnyak (Lemma 5.1). In higher dimensions the possibility of such an approximation is still not known even in the Euclidean case (see [4, page 59]).

Saddle surfaces in $\mathbb{S}_{\mathcal{K}}^{3}(\kappa \neq 0)$ can be defined in a similar way as in $\mathbb{E}^{3}$, that is, by means of the operation of cutting off crusts by $\kappa$-planes. Instead of this definition, we introduce an equivalent coordinate-free definition using only the geodesic structure of $\mathbb{S}_{\kappa}^{3}$.

In Section 2, we review the definition of a metric space of curvature bounded from above in the sense of Aleksandrov. In Section 3, we present the generalized definition of a saddle surface in an arbitrary geodesically connected space (Definition 3.1, Theorem 3.4). In Section 4, we determine the curvature condition that a saddle polyhedron in $\mathbb{S}_{\mathcal{K}}^{3}$ satisfies Proposition 4.4 and in Section 5, we give the proof of Theorem 1.1.

\section{Metric spaces of curvature bounded from above in the sense of Aleksandrov}

A notion of curvature of metric spaces can be defined by comparing triangles in a metric space with the corresponding model triangles in the $\kappa$-plane with sides of the same length. The definition is due to Aleksandrov [1] and the curvature is usually referred to as the curvature in the sense of Aleksandrov. Aleksandrov's spaces are a natural generalization of Riemannian manifolds but they are of much more general nature. For more details, see [2, 3].

An $\mathfrak{R}_{\kappa}$ domain, abbreviated by $\mathfrak{R}_{\kappa}$, is a metric space satisfying the following axioms.

Axiom 1. Any two points in $\Re_{\kappa}$ can be joined by a geodesic segment.

Axiom 2. If $\kappa>0$, then the perimeter of each triangle in $\Re_{\kappa}$ is less than $2 \pi / \sqrt{\kappa}$.

Axiom 3. Each triangle in $\mathfrak{R}_{\kappa}$ has nonpositive $\kappa$-excess, that is, for the angles $\alpha, \beta, \gamma$ of a triangle $A B C$

$$
\alpha+\beta+\gamma-\left(\alpha_{\kappa}+\beta_{\kappa}+\gamma_{\kappa}\right) \leq 0
$$


where $\alpha_{\kappa}, \beta_{\kappa}, \gamma_{\kappa}$ are the corresponding angles of a triangle $A^{\kappa} B^{\kappa} C^{\kappa}$ on the $\kappa$-plane with sides of the same length as $A B C$.

Another term for an $\mathfrak{R}_{\kappa}$ domain is a CAT $(\kappa)$ space. It is evident that any $\kappa$ space is an $\Re_{\kappa}$ domain. A space of curvature bounded by $\kappa$ from above in the sense of Aleksandrov is a metric space, each point of which is contained in some neighborhood of the original space, which is an $\Re_{\kappa}$ domain.

\section{Saddle surfaces}

Nonregular saddle surfaces in $\mathbb{E}^{n}$. A (parametrized) surface $f$ in $\mathbb{E}^{n}$ is any continuous mapping $f: D \rightarrow \mathbb{E}^{n}$, where $D$ denotes the closed unit disk on the plane. We say that a hyperplane $P$ with equation $a_{1} x_{1}+\cdots+a_{n} x_{n}=b$ cuts off a crust from the surface $f$ if among the connected components (maximal connected subsets) of $f^{-1}(f(D) \backslash P)$ there is one with positive distance from the boundary of $D$. It is clear that if $U$ is such a component, then $U$ is an open set and the set $f(U)$, which is called a crust, is contained in one of the two open half-spaces that the hyperplane $P$ defines. We always assume that $f(U) \subset P^{+}$and $f(\partial U) \subset P$, where $P^{+}$is the half-space determined by $a_{1} x_{1}+\cdots+a_{n} x_{n}>b$.

A surface $f$ in $\mathbb{E}^{n}$ is said to be a saddle surface if it is impossible to cut off a crust from it by any hyperplane (see [4]). Notice that saddle surfaces are, by definition, compact surfaces. The class of $C^{2}$ saddle surfaces in $\mathbb{E}^{3}$ coincides with the class of surfaces of nonpositive Gaussian curvature.

Nonregular saddle surfaces in metric spaces. Let $(M, d)$ be a geodesically connected metric space, and $D$ the closed unit disk on the plane. A (parametrized) surface $f$ in a metric space $M$ is any continuous mapping $f: D \rightarrow M$. The convex hull of a subset $A$, denoted by $\operatorname{conv}(A)$, is defined as the union of all sets $G^{(n)}(A)$, with $G^{(0)}(A)=A, G^{(1)}(A)$ is the union of all geodesic segments between points of $A$, and $G^{(n)}(A)=G^{(1)}\left(G^{(n-1)}(A)\right)$ for any $n>1$.

Definition 3.1. A surface $f$ in a geodesically connected space $M$ is said to be a saddle surface if

$$
f(\text { int } \gamma) \subset \operatorname{conv}(f(\gamma))
$$

for every Jordan curve $\gamma \subset D$ having positive distance from the unit circle.

Theorem 3.4 below shows the equivalence of Definition 3.1 with the classical one in the case of a Euclidean space. In order to prove it we need the following two elementary lemmas.

Lemma 3.2. Let $D_{1}, \ldots, D_{m}$ be closed disks in the plane such that $\bigcup_{i=1}^{m} D_{i}$ is a connected set. Then given an $\varepsilon>0$, there exists a Jordan plane curve $\gamma$ with the following properties: 
(a) $\gamma$ consists of a finite number of circular arcs each of which is a part of the boundary of some $D_{i}^{\prime}(i=1, \ldots, m)$, where $D_{i}^{\prime}$ is a closed disk with the same center as $D_{i}$ and its radius is $\left(1+\lambda_{i}\right)$ times the radius of $D_{i}$, where $0 \leq \lambda_{i}<\varepsilon$;

(b) $\bigcup_{i=1}^{m} D_{i} \subset \bigcup_{i=1}^{m} D_{i}^{\prime} \subset \overline{\operatorname{int} \gamma}$.

We sketch the proof of Lemma 3.2. The claim is obvious for $m=1$. Suppose that the claim is true for some $m \geq 1$. Let $\varepsilon>0$ and $D_{1}, \ldots, D_{m}, D_{m+1}$ be $m+$ 1 closed disks in the plane with $\bigcup_{i=1}^{m+1} D_{i}$ a connected set. We group the disks $D_{1}, \ldots, D_{m}$ into $k$ groups so that the union of each such group is a connected set. Then we apply the inductive assumption for each one of these groups and we get $k$ Jordan curves $\gamma_{1}, \ldots, \gamma_{k}$ and $m$ new closed disks $D_{1}^{\prime}, \ldots, D_{m}^{\prime}$. If $D_{m+1}$ touches any one of the disks $D_{1}^{\prime}, \ldots, D_{m}^{\prime}$, then we slightly enlarge $D_{m+1}$ to a new one $D_{m+1}^{\prime}$ that does not touch any one of them. Then the desired Jordan curve is the boundary of the unbounded component of $\mathbb{E}^{2} \backslash\left(\overline{\operatorname{int} \gamma_{1}} \cup \cdots \cup \overline{\operatorname{int} \gamma_{k}} \cup D_{m+1}^{\prime}\right)$.

Let $\delta>0$. The closure of a bounded connected set in the plane can be covered by a finite number of open disks of radius $\delta / 4$ the union of which is a connected set. Therefore, the Jordan plane curve that Lemma 3.2 ensures for the corresponding closed disks and for the positive number $\varepsilon=\delta / 4$ satisfies the two conditions of the following lemma.

Lemma 3.3. Let $U$ be a bounded, connected set in the plane and let $\delta$ be a positive number. Then there exists a Jordan plane curve $\gamma$ such that (i) $\gamma \subset \bigcup_{y \in \partial U} D(y, \delta)$, and (ii) $\bar{U} \subset$ int $\gamma$, where $D(y, \delta)$ denotes the open disk of radius $\delta$ centered at $y$.

The following theorem justifies our definition of a saddle surface.

Theorem 3.4. If $f$ is a surface in $\mathbb{E}^{n}$ then the following are equivalent:

(a) it is impossible to cut off a crust from $f$ by any hyperplane,

(b) $f(\operatorname{int} \gamma) \subset \operatorname{conv}(f(\gamma))$ for every Jordan curve $\gamma \subset D$ which has a positive distance from the unit circle.

Proof. (a) $\Rightarrow$ (b). Suppose, contrary to the claim, that there exist a Jordan curve $\gamma \subset D$ having a positive distance from the unit circle, and a point $a \in \operatorname{int}(\gamma)$ so that $f(a) \notin \operatorname{conv}(f(\gamma))$. We can separate the convex set $\operatorname{conv}(f(\gamma))$ from the point $f(a)$ by a hyperplane $P$ with $f(a) \in P^{+}$and $\operatorname{conv}(f(\gamma)) \subset P^{-}$, where $P^{+}$ and $P^{-}$are the two open half-spaces the hyperplane $P$ defines. If $V$ is the connected component of $f^{-1}\left(P^{+}\right)$that contains the point $a \in \operatorname{int}(\gamma)$, then $V$ does not intersect the curve $\gamma$ since $f(\gamma) \subset P^{-}$. So $V \subset \operatorname{int}(\gamma)$ and therefore the distance of $V$ from the unit circle is positive. Thus, the hyperplane $P$ cuts off a crust from $f$, a contradiction.

(b) $\Rightarrow$ (a). Suppose that a hyperplane $P$ cuts off a crust from $f$. Then $f(U) \subset$ $P^{+}$and $f(\partial U) \subset P$ for some open connected subset $U$ of $D$ having positive distance from the unit circle. Let $\varepsilon=\max \{\operatorname{dist}(x, P): x \in f(\bar{U})\}>0$. Since $f$ is a uniformly continuous function, there is a $\delta_{1}>0$ such that $f(D(y, \delta)) \subset$ $B(f(y), \varepsilon / 2)$ for all $\delta \in\left(0, \delta_{1}\right)$ with $D(y, \delta) \subset D$, where $B(f(y), \varepsilon / 2)$ denotes the 
$n$-dimensional ball of radius $\varepsilon / 2$ centered at $f(y)$. Since the distance of $U$ from the unit circle is positive, choose $\delta \in\left(0, \delta_{1}\right)$ so that $\bigcup_{y \in \partial U} D(y, \delta) \subset D$. Then

$$
\begin{gathered}
f(\gamma) \subset f\left(\bigcup_{y \in \partial U} D(y, \delta)\right) \subset \bigcup_{y \in \partial U} f(D(y, \delta)) \\
\subset \bigcup_{y \in \partial U} B\left(f(y), \frac{\varepsilon}{2}\right) \subset \bigcup_{z \in P} B\left(z, \frac{\varepsilon}{2}\right) .
\end{gathered}
$$

Therefore, $f(\gamma) \subset\{p+t n: p \in P$ and $-\varepsilon / 2 \leq t \leq \varepsilon / 2\}$, where $n$ is a unit normal vector to the hyperplane $P$. So $f($ int $\gamma) \subset \operatorname{conv}(f(\gamma)) \subset\{p+t n: p \in P$ and $-\varepsilon / 2 \leq$ $t \leq \varepsilon / 2\}$ therefore, by Lemma 3.3(ii), $f(\bar{U}) \subset\{p+t n: p \in P$ and $-\varepsilon / 2 \leq t \leq \varepsilon / 2\}$ which contradicts the choice of $\varepsilon$.

Definition 3.5. Let $M_{1}, M_{2}$ be two metric spaces. The mapping $\varphi: M_{1} \rightarrow M_{2}$ is called a geodesic mapping if the image of any geodesic segment in $M_{1}$ under $\varphi$ is a geodesic segment in $M_{2}$.

Example 3.6. For any $\kappa \in \mathbb{R}$ there exists a mapping $\varphi: \mathbb{S}_{\mathcal{K}}^{3} \rightarrow \mathbb{E}^{3}$ such that both $\varphi$ and $\varphi^{-1}$ are geodesic mappings.

Proof. The assertion is trivial when $\kappa=0$. When $\kappa<0$ consider the BeltramiKlein model of $\mathbb{H}_{\kappa}^{3}$. Since geodesic segments in the Beltrami-Klein model of $\mathbb{H}_{\mathcal{K}}^{3}$ coincide with the Euclidean line segments, the inclusion mapping $\varphi: \mathbb{H}_{\kappa}^{3} \rightarrow \mathbb{E}^{3}$ with $\varphi(x)=x$ and its inverse are geodesic mappings. In the case when $\kappa>0$ consider the central projection $\varphi: \mathbb{S}_{+}^{3}\left(\kappa^{-1 / 2}\right) \rightarrow \mathbb{E}^{3}$ defined by

$$
\varphi\left(x_{1}, x_{2}, x_{3}, x_{4}\right)=\kappa^{-1 / 2}\left(\frac{x_{1}}{x_{4}}, \frac{x_{2}}{x_{4}}, \frac{x_{3}}{x_{4}}\right) .
$$

The central projection takes a point $x$ on $\mathbb{S}_{+}^{3}\left(\kappa^{-1 / 2}\right)$ to the intersection of the hyperplane $\left\{x_{4}=\kappa^{-1 / 2}\right\} \equiv \mathbb{E}^{3}$ with the straight line through the point $x$ and the origin of $\mathbb{E}^{4}$. Under the mapping $\varphi$ great circles go to straight lines and vice versa. Therefore, both $\varphi$ and $\varphi^{-1}$ are geodesic mappings.

Proposition 3.7. Let $M_{1}, M_{2}$ be two metric spaces, $\varphi: M_{1} \rightarrow M_{2}$ be a geodesic mapping, and $f: D \rightarrow M_{1}$ be a saddle surface in $M_{1}$. Then $\varphi \circ f$ is a saddle surface in $M_{2}$.

Proof. It follows directly by the definition of saddle surfaces and convex hull.

\section{Curvature of saddle polyhedra in $\mathbb{S}_{\kappa}^{3}$}

In order to determine the curvature condition that saddle polyhedra in $\mathbb{S}_{\mathcal{K}}^{3}$ satisfy, we need to estimate the total angle at any point of such a polyhedron. All arguments in this section can be trivially generalized to higher dimensions.

A surface in $\mathbb{S}_{\kappa}^{3}$, defined over a domain in the Euclidean plane bounded by a simple closed polygonal line, is called a polyhedron if it can be partitioned into 
a finite number of $\kappa$-plane triangles intersected only at the boundaries. In order to estimate the total angle at a point of a saddle polyhedron in $\mathbb{S}_{\kappa}^{3}$, we need the following two lemmas.

Lemma 4.1. If $A_{1}, A_{2}, A_{3}, A_{4}$, and $O$ are points in $\mathbb{S}_{\kappa}^{3}$ such that $O$ belongs to the convex hull of $A_{1}, A_{2}, A_{3}$, and $A_{4}$, then

$$
\widehat{A_{1} O A_{2}}+\widehat{A_{2} O A_{3}}+\widehat{A_{3} O A_{4}}+\widehat{A_{4} O A_{1}} \geq 2 \pi \text {. }
$$

Proof. First let $\kappa=0 . O \in \operatorname{conv}\left\{A_{1}, A_{2}, A_{3}, A_{4}\right\}$ implies that there exists a point $D$ on the line segment $A_{3} A_{4}$ and a point $B$ on the line segment $A_{1} D$ such that $O$ lies on the line segment $A_{2} B$. Because of triangle inequality and since $A_{1}, A_{2}$, and $D$ are coplanar, we have

$$
\begin{aligned}
\widehat{A_{1} O A_{2}}+\widehat{A_{2} O A_{3}}+\widehat{A_{3} O A_{4}}+\widehat{A_{4} O A_{1}} \\
=\widehat{A_{1} O A_{2}}+\left(\widehat{A_{2} O A_{3}}+\widehat{A_{3} O D}\right)+\left(\widehat{D O A_{4}}+\widehat{A_{4} O A_{1}}\right) \\
\geq \widehat{A_{1} O A_{2}}+\widehat{A_{2} O D}+\widehat{D O A_{1}} \\
=2 \pi .
\end{aligned}
$$

Since in the Beltrami-Klein model of $\mathbb{H}_{\kappa}^{3}$ geodesic segments are Euclidean line segments, the proof in the hyperbolic case is exactly the same as in the Euclidean case. In the hemisphere $\mathbb{S}_{+}^{3}\left(\kappa^{-1 / 2}\right)$ we follow the same steps as in the Euclidean case. Equality (4.2) holds because the images of $A_{1}, A_{2}$, and $D$ under $\exp _{O}^{-1}$ are coplanar.

Lemma 4.2. Let $O, B$, and $A_{1}, A_{2}, \ldots, A_{k}$ be points in $\mathbb{S}_{\kappa}^{3}$. If $B$ belongs to the convex hull of $A_{1}, A_{2}, \ldots, A_{k}$, then

$$
\widehat{A O B}+\widehat{B O C} \leq \widehat{A O A_{1}}+{\widehat{A_{1} O A_{2}}}_{+\cdots+\widehat{A}_{k-1} O A_{k}}+\widehat{A_{k} O C}
$$

for any $A, C$ in $\mathbb{S}_{\kappa}^{3}$.

Proof. Apply induction on $k$ and the angle triangle inequality.

Proposition 4.3. The total angle at any point of a saddle polyhedron in $\mathbb{S}_{\kappa}^{3}$ is greater than or equal to $2 \pi$.

Proof. Let $O$ be a point on a saddle polyhedron in $\mathbb{S}_{\kappa}^{3}$. Then, by Definition 3.1, there are points $A_{1}, A_{2}, \ldots, A_{k}$ on the polyhedron such that $O \in \operatorname{conv}\left\{A_{1}\right.$, $\left.A_{2}, \ldots, A_{k}\right\}$. We will prove that

$$
\widehat{A_{1} O A_{2}}+{\widehat{A_{2} O A_{3}}}+\cdots+{\widehat{A_{k-1} O A_{k}}}_{k}+{\widehat{A_{k} O A_{1}}}^{2} 2 \pi
$$

If $k=3$ then relation (4.4) obviously holds as an equality. Let $k>3$, then there exists a point $B \in \operatorname{conv}\left\{A_{3}, \ldots, A_{k-1}\right\}$ such that $O \in \operatorname{conv}\left\{A_{1}, A_{2}, B, A_{k}\right\}$. By 
Lemma 4.2

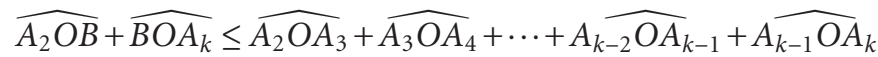

and, by Lemma 4.1,

$$
\widehat{A_{1} O A_{2}}+\widehat{A_{2} O B}+\widehat{B O A_{k}}+\widehat{A_{k} O A_{1}} \geq 2 \pi .
$$

Therefore,

$$
{\widehat{A_{1} O A_{2}}}+{\widehat{A_{2} O A_{3}}}+\cdots+{\widehat{A_{k-1} O A_{k}}}_{+\widehat{A k}_{k} O A_{1}} \geq 2 \pi
$$

Proposition 4.4. Any saddle polyhedron in a space of constant curvature $\kappa$ is a space of curvature bounded from above by $\kappa$ in the sense of Aleksandrov.

Proof. A necessary and sufficient condition for a locally geodesically connected space $M$ with intrinsic metric to be a space of curvature $\leq \kappa$ in the sense of Aleksandrov is

$$
\kappa_{\text {int }}(\mathfrak{p}) \leq \kappa \quad \forall \mathfrak{p} \in M
$$

with $\kappa_{\text {int }}(\mathfrak{p})$ to be the intrinsic curvature of $M$ at $\mathfrak{p}$, defined by

$$
\kappa_{\text {int }}(\mathfrak{p})=\varlimsup_{\mathscr{T} \rightarrow \mathfrak{p}} \frac{\delta(\mathscr{T})}{S(\mathscr{T})} .
$$

The limit is taken over all nondegenerate geodesic triangles $\mathscr{T}$ in $M$ the vertices of which approach the point $\mathfrak{p} . \delta(\mathscr{T})$ is the excess of $\mathscr{T}$, that is, $\delta(\mathscr{T})=\alpha+\beta+\gamma-\pi$ with $\alpha, \beta, \gamma$ the angles of $\mathscr{T}$, and $S(\mathscr{T})$ denotes the area of $\mathscr{T}$. This characterization of spaces of curvature bounded from above is due to Aleksandrov [1].

Let $P$ be a polyhedron in a space of constant curvature $\kappa$ and $\mathfrak{p}$ be a point on $P$. If the point $\mathfrak{p}$ is not a vertex, then by the Gauss-Bonnett formula,

$$
\delta(\mathscr{T})=\iint_{\mathscr{T}} \kappa d S=\kappa S(\mathscr{T})
$$

so $\kappa_{\text {int }}(\mathfrak{p})=\kappa$. Let $\mathfrak{p}$ be a vertex of $P$ which belongs to the interior of the triangle $\mathscr{T}$. Suppose that the edges of $P$, starting at the vertex $\mathfrak{p}$, intersect the sides of $\mathscr{T}$ into $N$ points. Joining $\mathfrak{p}$ with these $N$ points and the three vertices of the triangle $\mathscr{T}$, we can construct $N+3$ triangles each of which lies on only one face of $P$ with the singleton $\{\mathfrak{p}\}$ to be their intersection. Applying the Gauss-Bonnett formula to each of them, we have $\delta\left(\mathscr{T}_{1}\right)+\delta\left(\mathscr{T}_{2}\right)+\cdots+\delta\left(\mathscr{T}_{N+3}\right)=\kappa S(\mathscr{T})$ and therefore, if $\alpha, \beta, \gamma$ are the three angles of $\mathscr{T}$, then

$$
\alpha+\beta+\gamma-(N+3) \pi+[\text { total angle at } \mathfrak{p}]+N \pi=\kappa S(\mathscr{T}) .
$$

Hence, $\delta(\mathscr{T})=\kappa S(\mathscr{T})+[2 \pi-$ total angle at $\mathfrak{p}]$. But, by Proposition 4.3 , the total 
angle at any vertex of a saddle polyhedron is greater than or equal to $2 \pi$. Therefore $\delta(\mathscr{T}) \leq \kappa S(\mathscr{T})$, and hence $\kappa_{\text {int }}(\mathfrak{p}) \leq \kappa$.

\section{Curvature of saddle surfaces in $\mathbb{H}_{\kappa}^{3}$ and $\mathbb{S}_{+}^{3}\left(\kappa^{-1 / 2}\right)$}

In this section we prove Theorem 1.1. To do so we need the concept of a $\mathfrak{P}_{\kappa}$ domain and the following two lemmas.

A geodesically connected space with intrinsic metric is said to be a $\mathfrak{P}_{\kappa}$ domain if for any triangle contained in $\mathfrak{P}_{\kappa}$, whose perimeter is less than $2 \pi / \sqrt{\kappa}, \kappa>0$, the $\kappa$-excess is nonpositive. It is clear that $\mathfrak{P}_{\kappa}$ domains and $\mathfrak{R}_{\kappa}$ domains coincide in $\mathbb{S}_{\kappa}^{3}$.

Lemma 5.1 (see [6]). A geodesically connected space $M$ with intrinsic metric is a $\mathfrak{P}_{\kappa}$ domain if and only if for any closed rectifiable curve $\mathscr{L}$ in $M$ there exists a convex domain $V$ in $\mathbb{S}_{\kappa}^{2}$ with bounding curve $\mathcal{N}$ and a mapping $\varphi: V \rightarrow M$ such that (i) $\varphi$ is a nonexpanding mapping, that is, $d_{M}(\varphi(x), \varphi(y)) \leq d_{\mathbb{S}_{\kappa}^{2}}(x, y)$ for all $x, y \in V$ and (ii) $\varphi$ maps $\mathcal{N}$ onto $\mathscr{L}$ translating each arc of $\mathcal{N}$ onto an arc of $\mathscr{L}$ of the same length.

LemMA 5.2. Any nonparametric saddle surface in $\mathbb{S}_{\kappa}^{3}(\kappa \neq 0)$ can be approximated uniformly by a sequence of saddle polyhedra with the lengths of their bounding curves convergent to the length of the bounding curve of the saddle surface.

Proof. The case $\kappa=0$ is due to Shefel' $[4,7]$. Let $\kappa \neq 0$ and $\varphi$ the geodesic mapping from $\mathbb{S}_{\mathcal{K}}^{3}$ into $\mathbb{E}^{3}$ insured by Example 3.6. It is not difficult to see that the restriction of $\varphi: \mathbb{S}_{\kappa}^{3} \rightarrow \mathbb{E}^{3}$ to a compact set is a bi-Lipschitz mapping.

Comment 1 . Let $\left(g_{i j}(\kappa)\right)$ be the $3 \times 3$ positive definite symmetric matrix that the coefficients of the first fundamental form of $\mathbb{S}_{\kappa}^{3}$ define. Each $g_{i j}(\kappa)$ is a polynomial in $x_{1}, x_{2}, x_{3}$ depending on $\kappa$. Assume that $\lambda_{1}$ and $\lambda_{2}$ are the minimum and maximum eigenvalue of $\left(g_{i j}(\kappa)\right)$, respectively. Then, since $\varphi$ is restricted on a compact set, there are positive constants $k_{1}, k_{2}$ such that $0<k_{1} \leq \lambda_{1} \leq \lambda_{2} \leq k_{2}$ and

$$
k_{1} \leq \frac{\sum_{i j=1}^{3} g_{i j} d x_{i} d x_{j}}{d x_{1}^{2}+d x_{2}^{2}+d x_{3}^{2}} \leq k_{2}
$$

that is,

$$
k_{1}\left(d x_{1}^{2}+d x_{2}^{2}+d x_{3}^{2}\right) \leq d s_{\mathbb{S}_{\kappa}^{3}}^{2} \leq k_{2}\left(d x_{1}^{2}+d x_{2}^{2}+d x_{3}^{2}\right) .
$$

Equation (5.2) completes the proof of our assertion for $\kappa<0$. Let $\kappa>0$. On a compact subset of $x_{1}^{2}+x_{2}^{2}+x_{3}^{2}<1 / \kappa$ the element of length $d s^{2}$ of the coordinate system (3.3), where $x_{4}=\left(1 / \kappa-x_{1}^{2}-x_{2}^{2}-x_{3}^{2}\right)^{1 / 2}$, satisfies the inequality

$$
c_{1}\left(d x_{1}^{2}+d x_{2}^{2}+d x_{3}^{2}\right) \leq d s^{2} \leq c_{2}\left(d x_{1}^{2}+d x_{2}^{2}+d x_{3}^{2}\right)
$$

for some positive constants $c_{1}, c_{2}$. Therefore, for any $\kappa \neq 0$, the restriction of $\varphi: \mathbb{S}_{\kappa}^{3} \rightarrow \mathbb{E}^{3}$ to a compact set is a bi-Lipschitz mapping. 
We are now ready to complete the proof of the lemma.

Let $f$ be a nonparametric saddle surface in $\mathbb{S}_{\kappa}^{3}$ and let $\left\{P_{n}: n \in \mathbb{N}\right\}$ be the sequence of Euclidean saddle polyhedra approximating the nonparamertic saddle surface $\varphi(f)$. Then, $\varphi^{-1}\left(P_{n}\right)$ is the desired sequence.

Remark 5.3. The fact that the geodesic mapping $\varphi: \mathbb{S}_{\kappa}^{3} \rightarrow \mathbb{E}^{3}$ is bi-Lipschitz on the compact sets has two important consequences; there are positive constants $k_{1}, k_{2}$ depending on the compact set such that for any curve $\gamma$ and surface $f$ in the compact set $k_{2} \ell(\gamma) \leq \ell(\varphi \circ \gamma) \leq k_{1} \ell(\gamma)$ and $k_{2}^{2} S(f) \leq S(\varphi \circ f) \leq k_{1}^{2} S(f)$, where $\ell$ denotes length and $S$ denotes the Lebesgue area (see [5]).

It is a well-known property of a two-dimensional one connected Euclidean surface with nonpositive curvature that its intrinsic diameter does not exceed the half of the length of its bounding curve. Hence, by Proposition 4.4, Remark 5.3, and Lemma 5.2 it follows that any pair of points on the graph $\mathscr{S}$ of a nonparametric surface in $\mathbb{S}_{\mathcal{\kappa}}^{3}$ can be joint by a rectifiable curve on $\mathscr{S}$. Therefore, if we consider $\mathscr{S}$ as a metric space with distance between two points the minimum length of the curves lying on $\mathscr{S}$ and joining those points, then $\mathscr{S}$ is a space with an intrinsic metric.

Proof of Theorem 1.1. Let $\mathscr{S}$ be the graph of a nonparametric saddle surface in $\mathbb{S}_{\mathcal{K}}^{3}$ bounded by a rectifiable curve. To show that $\mathscr{S}$ is a space of curvature not greater than $\kappa$ in the sense of Aleksandrov it suffices to prove that for any curve $\mathscr{L}$ on $\mathscr{S}$ of length $\ell$ there exists a nonexpanding mapping $\varphi$ as described in Lemma 5.1. Let $W$ be the neighborhood on $\mathscr{S}$ with boundary curve a given curve $\mathscr{L}$ of length $\ell$. Consider $W$ as a space with intrinsic metric induced by the metric of $\mathbb{S}_{\kappa}^{3}$. Construct a sequence of saddle polyhedra $P_{n}$ convergent to $W$ uniformly, so that if $\ell_{n}$ is the length of the boundary curve $\mathscr{L}_{n}$ of $P_{n}$ then $\lim _{n \rightarrow \infty} \ell_{n}=\ell$. By Proposition 4.4 each $P_{n}$, as a space with intrinsic metric, is a space of curvature bounded from above by $\kappa$ in the sense of Aleksandrov. For the boundary curve $\mathscr{L}_{n}$ of any saddle polyhedron $P_{n}$ construct, using Lemma 5.1, a nonexpanding mapping $\varphi_{n}: V_{n} \rightarrow P_{n}$ such that (a) $d_{n}\left(\varphi_{n}(x), \varphi_{n}(y)\right) \leq d_{\mathbb{S}_{\kappa}^{2}}(x, y)$ for all $x, y \in V_{n}$, and (b) $\varphi_{n}$ maps $\mathcal{N}_{n}$ onto $\mathscr{L}_{n}$ translating each arc of $\mathcal{N}_{n}$ onto an arc of $\mathscr{L}_{n}$ of the same length, where $V_{n}$ is a convex domain in $\mathbb{S}_{\kappa}^{2}$ with bounding curve $\mathcal{N}_{n}$, and $d_{n}$ is the intrinsic metric of $P_{n}$. Since the lengths of $\mathcal{N}_{n}$ are uniformly bounded we can assume, without loss of generality, that the sequence of convex domains $V_{n}$ converges to a convex domain $V$ with bounding curve $\mathcal{N}$ in the Hausdorff sense. The mapping $\varphi: V \rightarrow W$ defined by $\varphi(x)=\lim _{n \rightarrow \infty} \varphi_{n}\left(x_{n}\right)$, where $\left\{x_{n} \in V_{n}: n=1,2, \ldots\right\}$ is a sequence convergent to $x$, is a well-defined mapping because

$$
d_{n}\left(\varphi_{n}\left(x_{n}\right), \varphi_{n}\left(y_{n}\right)\right) \leq d_{\mathbb{S}_{\kappa}^{2}}\left(x_{n}, y_{n}\right) \quad \forall n=1,2, \ldots
$$

Taking liminf on both sides of the above inequality and using the semi-continuity of length, we have that $\varphi$ is a nonexpanding mapping. Condition (b) and the 
choice of $V$ and $\mathscr{L}_{n}$ imply that $\varphi$ maps $\mathcal{N}$ onto $\mathscr{L}$ translating each $\operatorname{arc}$ of $\mathcal{N}$ onto an $\operatorname{arc}$ of $\mathscr{L}$ of the same length. This completes the proof of Theorem 1.1.

\section{Remarks}

(1) The curvature condition in Theorem 1.1 is a necessary but not sufficient condition for a nonparametric surface with rectifiable bounding curve to be saddle, as the following elementary example indicates. Similar examples in $\mathbb{H}_{\kappa}^{3}$ and $\mathbb{S}_{+}^{3}\left(\kappa^{-1 / 2}\right)$ can be obtained by considering the geodesic mappings of Example 3.6.

Example 6.1. Consider the polyhedron $P$ defined by the points $A_{1}(0,0,0)$, $A_{2}(1,0, \varepsilon), A_{3}(0,0,1), A_{4}(0,1, \varepsilon), A_{5}(-1,0, \varepsilon)$, and $A_{6}(0,-1, \varepsilon)$, where $\varepsilon$ is any sufficiently small positive number. The bounding curve of $P$ is the polygonal line $A_{2} A_{3} A_{4} A_{5} A_{6} A_{2}$ and the only vertex is the point $A_{1}$. If $\theta(\varepsilon)$ is the total angle of $P$ at the vertex $A_{1}$, then $\lim _{\varepsilon \rightarrow 0} \theta(\varepsilon)=5 \pi / 2>2 \pi$. The intrinsic curvature of $P$ is, by definition, zero everywhere except the vertex $A_{1}$ where it is equal to $2 \pi-\theta(\varepsilon)$. Therefore, for sufficiently small $\varepsilon>0$ the intrinsic curvature of $P$ is nonpositive. But on the other hand, for any such $\varepsilon$ the polyhedron $P$ is not a saddle since we can cut off a crust about the vertex $A_{1}$.

(2) In [8] it is proved that any simply connected saddle surface in $\mathbb{E}^{3}$ satisfies the isoperimetric inequality $\alpha S-\ell^{2} \leq 0$ for some positive constant $\alpha$. Therefore, by Remark 5.3 , any simply connected saddle surface in $\mathbb{S}_{\mathcal{K}}^{3}$ satisfies the isoperimetric inequality $\beta S-\ell^{2} \leq 0$ for some positive constant $\beta$ depending on the distance of the surface from the boundary of the space. Hence, any simply connected saddle surface in $\mathbb{S}_{\mathcal{K}}^{3}$ with rectifiable bounding curve has finite area. On the other hand, in [5] it is proved that at each point of a surface in $\mathbb{E}^{3}$ with finite Lebesgue area there are arbitrarily small neighborhoods bounded by rectifiable curves. By Remark 5.3, this is also true in any space of constant curvature. Therefore, Theorem 1.1 can be strengthened as follows.

THEOREM 6.2. If a saddle surface in $\mathbb{S}_{\kappa}^{3}(\kappa \neq 0)$ has a rectifiable bounding curve, and in a neighborhood of each of its points it is nonparameric, then it is a space of curvature bounded from above by $\kappa$ in the sense of Aleksandrov.

(3) Since any simply connected saddle surface in $\mathbb{E}^{2}$ can be approximated by a sequence of saddle polyhedra (see [8]), one can easily derive the following theorem by applying arguments similar to what we used in the proof of Theorem 1.1.

Theorem 6.3. Any simply connected saddle surface in $\mathbb{S}_{\kappa}^{2}(\kappa \neq 0)$ has a curvature not greater than $\kappa$ in the sense of $A$. D. Aleksandrov.

\section{Acknowledgments}

The author expresses his gratitude to Professor Igor G. Nikolaev for his useful discussions on this paper. This work was partially supported by the Alexander S. Onassis Public Benefit Foundation. 


\section{References}

[1] A. D. Alexandrov, Über eine Verallgemeinerung der Riemannschen Geometrie, Schr. Forschungsinst. Math. 1 (1957), 33-84.

[2] W. Ballman, Lectures on Spaces of Nonpositive Curvature, DMV Seminar, vol. 25, Birkhäuser Verlag, Basel, 1995.

[3] V. N. Berestovskij and I. G. Nikolaev, Multidimensional generalized Riemannian spaces, Geometry, IV, Encyclopaedia of Math. Sciences, vol. 70, Springer, Berlin, 1993, pp. 165-243, 245-250.

[4] Yu. D. Burago and S. Z. Shefel', The geometry of surfaces in Euclidean spaces, Geometry, III, Encycl. Math. Sci., vol. 48, Springer, Berlin, 1992, pp. 1-85.

[5] L. Cesari, Surface Area, Annals of Mathematics Studies, vol. 35, Princeton University Press, New Jersy, 1956.

[6] Yu. G. Reshetnyak, Nonexpanding mappings in a space of curvature not greater than $K$, Siberian Math. J. 9 (1968), 683-689, translated from Sibirsk. Mat. Zh. 9 (1989), no. 4, 918-927.

[7] S. Z. Shefel', On the intrinsic geometry of saddle surfaces, Sibirsk. Mat. Ž. 5 (1964), 1382-1396.

[8] _ On saddle surfaces bounded by a rectifiable curve, Dokl. Akad. Nauk SSSR 162 (1965), 294-296, translated from Dokl. Akad. Nauk SSSR 162 (1965), 294296.

Dimitrios E. Kalikakis: Department of Mathematics, University of Illinois at Urbana-Champaign, 1409 West Green Street, Urbana, IL 61801, USA

Current address: Department of Applied Mathematics, University of Crete, Heraklion, 714-09, Crete, Greece

E-mail address: kalikak@tem.uoc.gr 


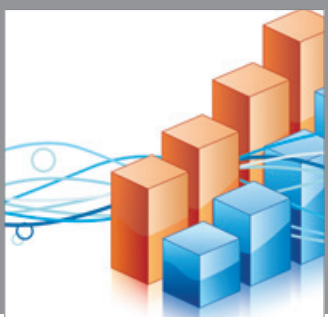

Advances in

Operations Research

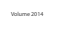

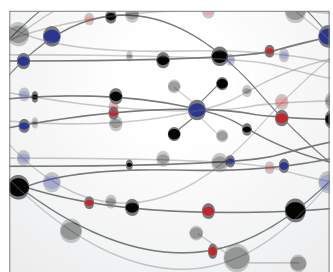

\section{The Scientific} World Journal
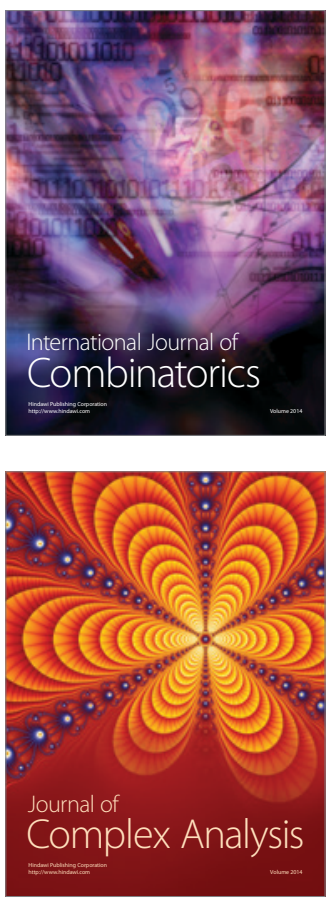

International Journal of

Mathematics and

Mathematical

Sciences
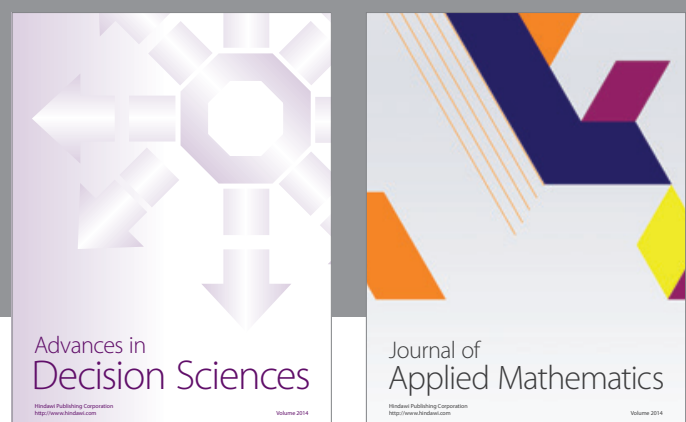

Journal of

Applied Mathematics
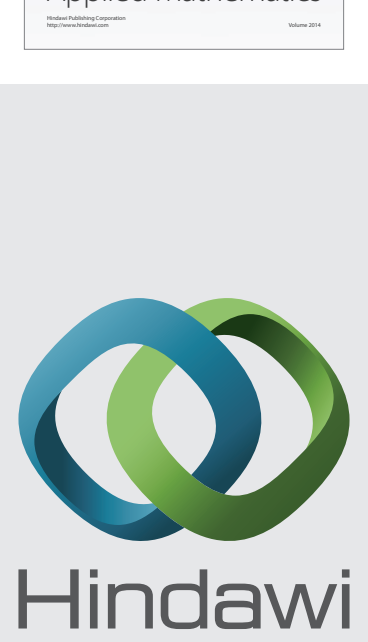

Submit your manuscripts at http://www.hindawi.com
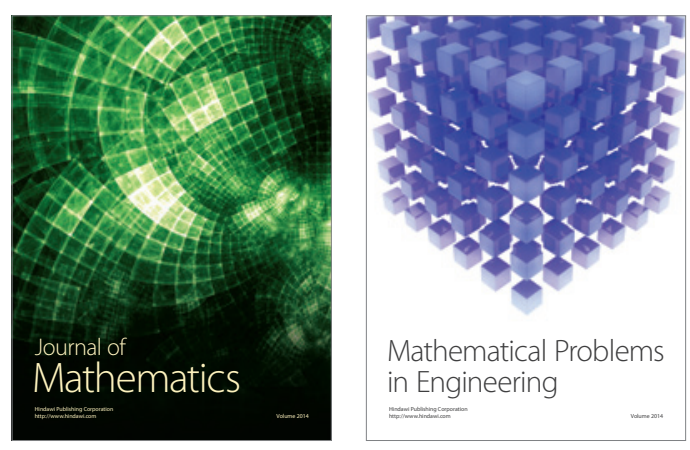

Mathematical Problems in Engineering
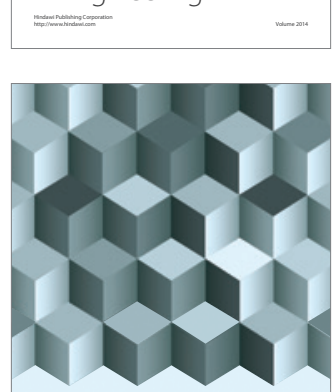

Journal of

Function Spaces
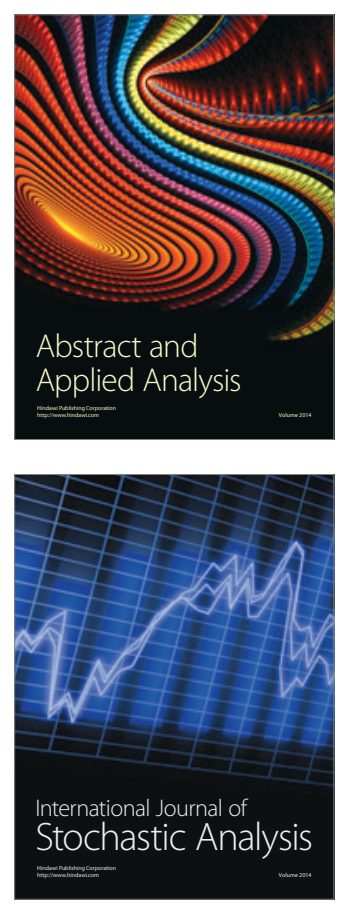

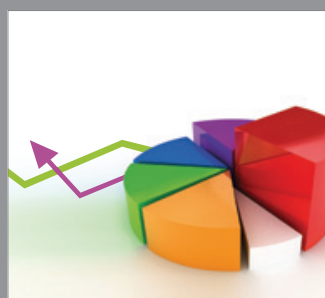

ournal of

Probability and Statistics

Promensencen
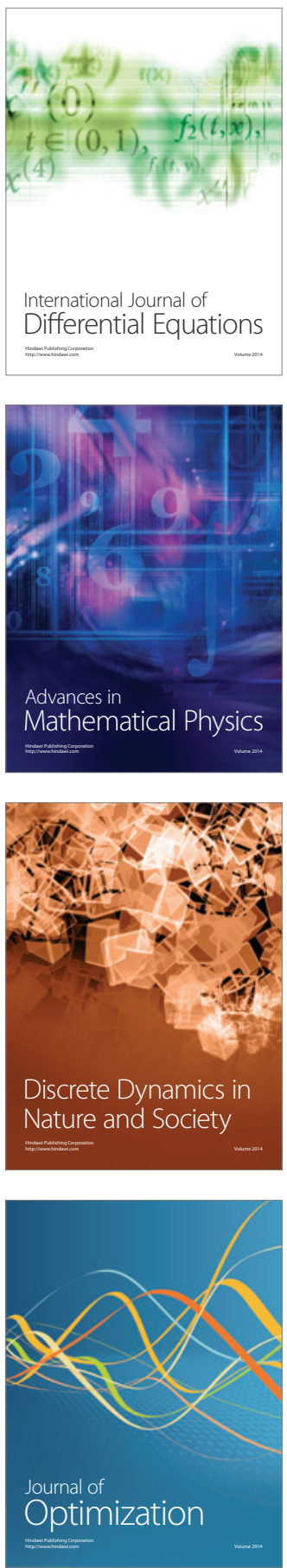